\title{
Elaboración de biocombustibles sólidos densificados a partir de tusa de maíz, bioaglomerante de yuca y carbón mineral del departamento de Córdoba
}

\author{
Development of densified solid biofuels a from corn cob, cassava starch \\ and mineral coal department of Córdoba
}

\author{
Cristian Berastegui Barranco ${ }^{1} \quad$ Juan Pablo Ortega Rodríguez ${ }^{1}$ \\ Jorge Mario Mendoza Fandiño ${ }^{1 *} \quad$ Yahir Enrique González Doria ${ }^{1}$ \\ Rafael David Gómez Vasquez² \\ Recibido 18 de abril de 2016, aceptado 21 de noviembre de 2016 \\ Received: April 18, 2016 Accepted: November 21, 2016
}

\begin{abstract}
RESUMEN
El departamento de Córdoba, cuenta con 45.000 toneladas de residuo de maíz (tusa de maíz) al año, que no representan ningún tipo de valor agregado al productor y son quemados a cielo abierto, generando un problema más de contaminación. Sin embargo, esta biomasa residual, puede ser utilizada como combustible mediante procesos de densificación. El objetivo de esta investigación fue establecer la mezcla óptima de tusa de maíz, carbón mineral con alto porcentaje de azufre producido en la Mina la Guacamaya de Puerto Libertador (Córdoba-Colombia) y aglomerante de yuca realizando un diseño experimental de mezclas simplex reticular, con el fin de obtener briquetas con buena resistencia a la compresión y durabilidad según las normas CEN/TS:15210 parte dos y NTC-2060, respectivamente. Además de incrementar el poder calorífico de la biomasa adicionando carbón mineral sin que se aumente significativamente el porcentaje de azufre. Se obtuvieron briquetas con resistencia a la compresión de $223,7 \mathrm{~kg}_{\mathrm{f}}$, durabilidad de $97,3 \%$, poder calorífico de $17,5 \mathrm{MJ} / \mathrm{kg}$ y un porcentaje de azufre de $0,4 \%$.
\end{abstract}

Palabras clave: Biocombustibles, durabilidad, poder calorífico, briquetas, bioaglomerante.

\begin{abstract}
The department of Córdoba produces 45,000 tons of waste from corn (corncob) per year, which does not represent any added value to the producer. Also, the residues are burnt in open air, producing atmospheric pollution issues. However, this residual biomass can be used as fuel by densification processes. The objective of this research was to establish the optimal mixture of corncob, coal with a high percentage of sulfur produced in Mina the Guacamaya Puerto Libertador (Córdoba-Colombia) and binder cassava through a statistical analysis of an experimental design of reticular simplex mixtures, in order to obtain briquettes with good compressive strength and durability according to CEN / standards TS: 15210 part two and NTC-2060 respectively. Furthermore, in this investigation the heating value of coal is raised by adding biomass. However, this addition does not significantly increase the percentage of sulfur. The briquettes were obtained with a compressive strength of $223.7 \mathrm{kgf}$, durability $97.3 \%, 17.5$ calorific value $M J / \mathrm{kg}$ and a percentage of $0.4 \%$ sulfur.
\end{abstract}

Keywords: Biofuels, durability, calorific value, briquettes, bio-binder.

1 Departamento Ingeniería Mecánica. Universidad de Córdoba. Carrera 6 \# 76-103. Montería, Colombia. E-mail: cberasb@gmail.com; juanpax1993@gmail.com; jorgemmf@gmail.com; yahirgonzalez@correo.unicordoba.edu.co

2 Facultad de Ingeniería Mecánica. Universidad Pontificia Bolivariana. Carrera 6 No 97ª-99. Montería, Colombia. E-mail: rafaeldavid11@gmail.com

* Autor de correspondencia. 


\section{INTRODUCCIÓN}

En el departamento de Córdoba la agricultura es una de las principales actividades económicas, este cuenta con un total de 103.007 hectáreas de tierra dedicadas a esta actividad, de las que 43.979 son utilizadas para la siembra de Maíz con una producción anual aproximada de 154.273 toneladas, cubriendo un $14 \%$ del total de la producción nacional [1]. Estos cultivos generan residuos que se han convertido en una fuente de contaminación, considerando que usualmente son quemados a cielo abierto, sumado al hecho de que no generan ningún tipo de valor agregado para el productor y presentan sobrecostos de manejo. Además, si se piensa en la utilización de estos residuos agrícolas en estado natural con fines energéticos, debido a su baja densidad se hace necesario grandes volúmenes de almacén y sus desiguales características morfológicas encarecen notablemente su utilización [2]. Por tanto, es de suma importancia que estos residuos tengan un proceso que mejore sus características energéticas, manipulación, transporte y almacenamiento. De esta manera, merece especial atención el uso de las biomasas residuales como biocombustibles sólidos, mediante la aplicación de procesos de densificación como empaquetado, cubicación, granulación o la fabricación de briquetas, los que mejoran de forma sustancial la densidad energética de la biomasa [3], simplifican la logística de manejo y almacenamiento, puesto que tienen forma y tamaño definido, tienen bajo contenido de polvo (seguridad durante la manipulación), son secas y pueden ser almacenadas sin degradar [4].

Las propiedades energéticas de la tusa de maíz en cuanto a combustible sólido, son inferiores a las del carbón sub-bituminoso producido en la mina la Guacamaya de Puerto Libertador (CórdobaColombia), ya que este tiene un poder calorífico de aproximadamente $5.715 \mathrm{Cal} / \mathrm{g}$, mientras que la tusa de maíz tiene un poder calorífico promedio de $3.792 \mathrm{Cal} / \mathrm{g}$ en base seca. Este tipo de carbón está caracterizado por tener un porcentaje de azufre de $1,55 \%$, lo que hace que sea poco apetecido en la industria siderúrgica y acería, uno de los mayores clientes industriales para el mercado del carbón ya que el Ministerio del Medio Ambiente del gobierno colombiano permite que este porcentaje sea máximo de $1 \%$, a diferencia del carbón producido en los yacimientos del Cerrejón (Guajira-Colombia), que tiene muy buena aceptación debido a que este posee un porcentaje de azufre y poder calorífico de $0,43 \%$ y $6,440 \mathrm{Cal} / \mathrm{g}$. respectivamente.

Es ideal conocer los porcentajes en peso de biomasa y aditivos empleados; así como la humedad a la que se manipulan estos productos. Conocidos estos porcentajes puede evaluarse de forma aproximada la composición química de briquetas. El poder calorífico de las briquetas será función del material de procedencia. Suponiendo que es madera sin aditivos su poder calorífico será el de la madera que proviene [5].

En investigaciones realizadas se concluye que recientemente en Polonia, así como en el resto de la Unión Europea (UE), se han utilizado cada vez más a menudo fuentes renovables en la generación de energía. En 2009, la energía procedente de fuentes renovables en la UE representó el 18,3\% del total de energía primaria, de la que la mayoría se obtiene a partir de biomasa sólida [6].

Se han realizado investigaciones en la determinación y comparación de durabilidad mecánica de briquetas producidas a partir de cultivos energéticos seleccionados, como son la biomasa de alto rendimiento anual y cultivos de plantas perennes, como el miscanthus, carrizo, knotweed gigante, el sorgo dulce y el cáñamo industrial, que pueden ser una fuente de energía renovable en los ámbitos adecuados. Se hicieron briquetas en estados puros y mezclados con biomasa de madera, para un total de veinte tipos de briquetas producidas por prensa hidráulica de pistón y su durabilidad mecánica se determinó de acuerdo con las normas europeas de biocombustibles sólidos. La investigación dio como resultado que la durabilidad de este tipo de briquetas se puede aumentar con la adición de residuos de madera y que el contenido de humedad del material debe ser tenido en cuenta en el proceso de fabricación, ya que tiene un gran impacto en la calidad final de las briquetas producidas [7].

Por otra parte, la combustión de los pellets y briquetas es más atractiva ambientalmente, debido a que reduce las emanaciones de $\mathrm{CO}_{2}$ en un $50 \%$ comparado con la combustión de leña o astillas, posee bajas concentraciones de azufre y nitrógeno entre $0,004-0,007 \%$ y $0,05-0,16 \%$ del peso seco final de cada pellet respectivamente. En consecuencia 
la combustión de briquetas contribuye a disminuir las concentraciones de $\mathrm{CO}_{2}$, óxidos de azufre y nitrógeno causantes del "efecto invernadero" y el cambio climático [8].

Se examinó las propiedades de briquetas producidas a partir de tusa de maíz y cascarilla de arroz, para establecer cuál de los dos residuos puede ser usado más eficiente y racionalmente como combustible [9]. De esta manera, se encontró que se pueden elaborar buenos biocombustibles sólidos con las dos biomasas, no obstante, las briquetas de tusa de maíz tuvieron mejores resultados, entre los que se destaca, un poder calorífico de $20,89 \mathrm{MJ} / \mathrm{Kg}$ y una resistencia a la compresión de $2,34 \mathrm{KN} / \mathrm{m}^{2}$, respecto a la cascarilla de arroz que obtuvo $13,39 \mathrm{MJ} / \mathrm{Kg}$ y $1,07 \mathrm{KN} / \mathrm{m}^{2}$, respectivamente. La durabilidad y la densidad de las partículas son los principales parámetros que describen la calidad física de los biocombustibles sólidos densificados como pellets y briquetas [10].

La influencia de la presión, la temperatura, la humedad y el tamaño de partícula sobre las propiedades físicas como la densidad, durabilidad, resistencia a la compresión y resistencia al impacto de briquetas hechas a partir de biomasa residual de Caragana korshinskii Kom, mediante un análisis estadístico multivariable. Obteniendo que el factor más influyente en las propiedades físicas de las briquetas es el tamaño de partícula, seguido por el contenido de humedad y la temperatura [11]. Así, se pueden producir briquetas de buena calidad usando un tamaño de partícula reducido entre 0,16 y $0,63 \mathrm{~mm}$, un bajo contenido de humedad (8-14\%) y condiciones de alta temperatura $\left(90-150^{\circ} \mathrm{C}\right)$. La presión no fue un factor relevante en el experimento. Por otra parte, se ha determinado la posibilidad de producir briquetas duraderas con paja de trigo y aserrín, como combustible para los hogares y pequeñas industrias de Kenia. Se determinó la expansión longitudinal de las briquetas en intervalos de tiempo específicos durante un período total de una semana, además el contenido de humedad y la durabilidad de estas briquetas se midieron después de un período de almacenamiento de dos semanas a aproximadamente $20^{\circ} \mathrm{C}$ y humedad relativa del $50 \%$. Se obtuvo que las briquetas de aserrín fueron más duraderas con un valor promedio de $88,4 \%$ y exhibieron un menor grado de expansión longitudinal de $70 \%$, mientras que aquellas hechas con paja de trigo mostraron una menor durabilidad con un promedio de 46,5\%, y un mayor grado de expansión de $150 \%$ en aumento; sin embargo, se encontró también que la mezcla de aserrín y paja de trigo con igualdad de fracciones en la mezcla mejoran considerablemente la durabilidad, respecto a los resultados con paja de trigo, presentando un aumento de $10 \%$. Parece, entonces, que hay una relación directa entre la expansión de longitud y la durabilidad de las briquetas [12].

El objetivo de este proyecto fue aprovechar la biomasa residual en cultivos de maíz del departamento de Córdoba, mediante la elaboración de briquetas con aglomerante de yuca y carbón mineral de la mina la Guacamaya, generando una alternativa a la utilización del carbón y buscando que este disminuyera su porcentaje de azufre y aumentara el poder calorífico de la briqueta, mejorando las características energéticas del densificado, que junto a la evaluación de las propiedades como la durabilidad y resistencia a la compresión, fueron los criterios para definir las mezclas óptimas, que presentan el mejor comportamiento frente a procesos de manipulación y transporte.

\section{ANÁLISIS TEÓRICO}

Para la realización de las briquetas primero fue necesario preparar la biomasa y el carbón realizando un proceso de molienda con un molino de martillo con una potencia nominal de $10 \mathrm{HP}$ como se observa en la Figura 1. Se caracterizó la granulometría de las biomasas y el carbón por medio de un proceso de tamizado, utilizando el ensayo U.S.A Standard Testing Siere, regido por la norma ASME E-11/2004. Con este ensayo se buscó determinar rangos en el tamaño de partícula del material ya triturado (distribución granulométrica), teniendo en cuenta que para la producción de briquetas, el óptimo debe ser inferior a $3 \mathrm{~mm}$, con el fin de garantizar la compactación y el acabado superficial [13].

Una vez terminado el proceso de molienda se realizó un diseño de experimento, con el fin de determinar el número de corridas y las proporciones de cada mezcla, para esto se estableció el porcentaje de biomasa, porcentaje de aglomerante y porcentaje de carbón como los factores y la durabilidad, resistencia a la compresión, poder calorífico y porcentaje de azufre como las variables de respuesta. Se escogió 

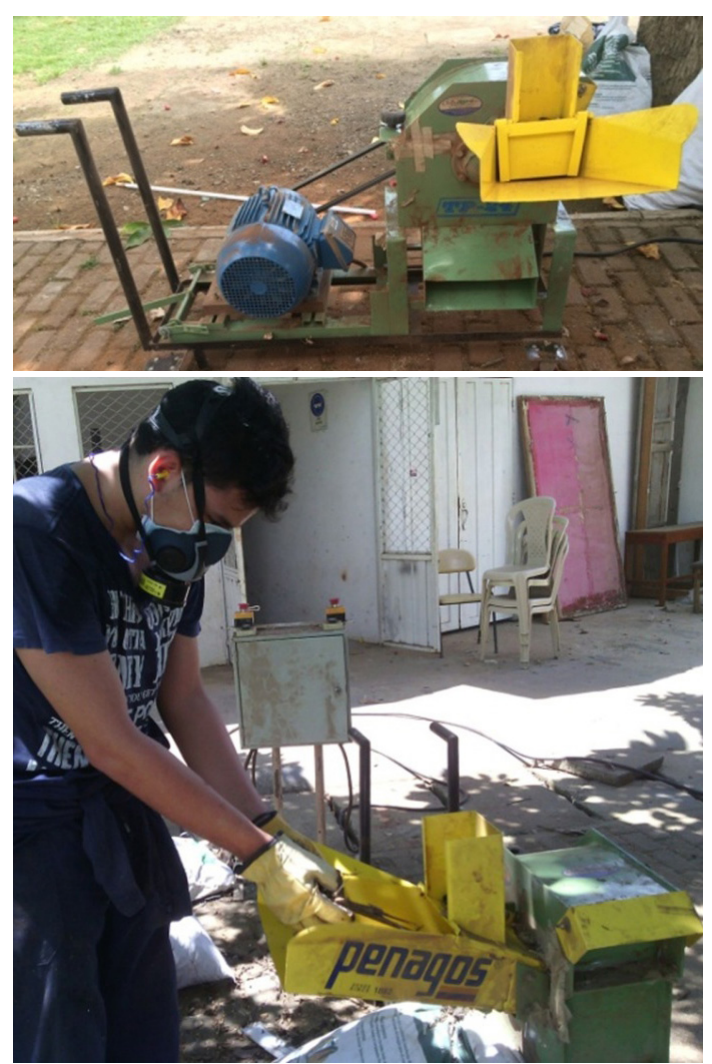

Figura 1. Molino de martillo.

Fuente: Autores (2015). Registro fotográfico.

un modelo simplex reticular debido a que este es un diseño experimental típico para mezclas de tres componentes, por otra parte se definieron los límites de los factores, teniendo en cuenta el uso racional del aglomerante, ya que porcentajes altos de este, influenciarían de manera directa en el costo, complejidad y poder calorífico de la briqueta. Por otra parte, con la inclusión del carbón en la mezcla se buscó aprovechar la energía potencial de este sin afectar de sobremanera el ambiente, debido a su alto contenido de azufre, por tanto se trabajó con rangos medios en el contenido de carbón de la mezcla para así limitar el azufre presente en las briquetas. De igual forma, la biomasa debe ser el factor que prime en la mezcla, por ello representa los valores superiores en los porcentajes (ver Tabla 1).

El proceso de briquetado se realizó a una temperatura ambiente promedio de $30^{\circ} \mathrm{C}$, elaborando 4 réplicas por cada mezcla del diseño experimental, de las que 2 se utilizaron para prueba de resistencia a la
Tabla 1. Límites de los porcentajes.

\begin{tabular}{|l|c|c|c|}
\hline \multicolumn{1}{|c|}{ Factores } & Unidades & $\begin{array}{c}\text { Valor } \\
\text { mínimo }\end{array}$ & $\begin{array}{c}\text { Valor } \\
\text { máximo }\end{array}$ \\
\hline Biomasa & $\%$ & 40 & 95 \\
\hline Aglomerante & $\%$ & 5 & 20 \\
\hline Carbón mineral & $\%$ & 0 & 40 \\
\hline
\end{tabular}

Fuente: Autores (2015).

compresión y 2 para prueba de durabilidad debido a los requerimientos de peso según la norma CEN/TS:15210 parte dos. Cada briqueta se hizo considerando una masa de $50 \mathrm{~g}$ y un diámetro fijo en la máquina de $52 \mathrm{~mm}$, obteniéndose una longitud de $80 \mathrm{~mm}$, teniendo en cuenta que esta longitud tuvo que ser inferior a dos veces el diámetro, para evitar efectos de pandeo en las briquetas. La presión de compactación fue de 7 $\mathrm{MPa}$, con un tiempo de sostenimiento de la carga de 10 minutos, la briqueta fue retirada del equipo después de 3 minutos, con el fin de contrarrestar los efectos de expansión volumétrica tras liberar a la briqueta de la presión aplicada como se observa en la Figura 2.

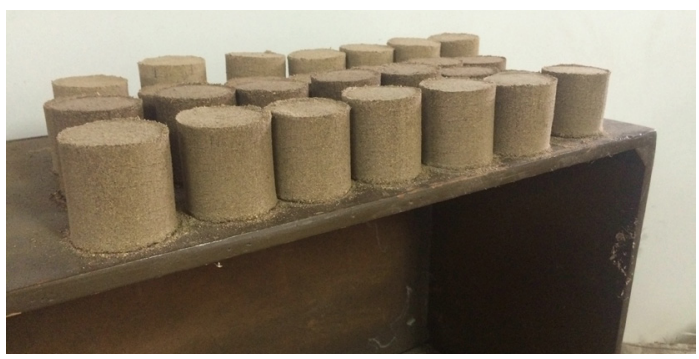

Figura 2. Briquetas de tusa de maíz.

Fuente: Autores (2015). Registro fotográfico.

Las briquetas fueron sometidas a pruebas de resistencia a la compresión y durabilidad, con el fin de medir la capacidad de estas a permanecer intactas frente a procesos de manipulación y transporte. El ensayo de resistencia a la compresión se realizó utilizando una máquina de compresión inconfinada con una capacidad máxima de 223 $\mathrm{kg}_{\mathrm{f}}$ ilustrado en la Figura 3, aplicando la carga hasta la falla de la briqueta. Los resultados en la prueba, se definieron a partir del requerimiento de resistencia al aplastamiento que debe tener una 
briqueta combustible, establecido en el numeral 4.2.1 de la norma técnica colombiana NTC-2060.

Por otra parte, la prueba de durabilidad se desarrolló bajo la norma CEN/TS 15210 parte dos para briquetas, en la que se sometieron a golpes controlados entre sí y contra las paredes de una cámara rotativa que gira a una velocidad de 21 RPM durante 5 minutos como se observa en la Figura 4.

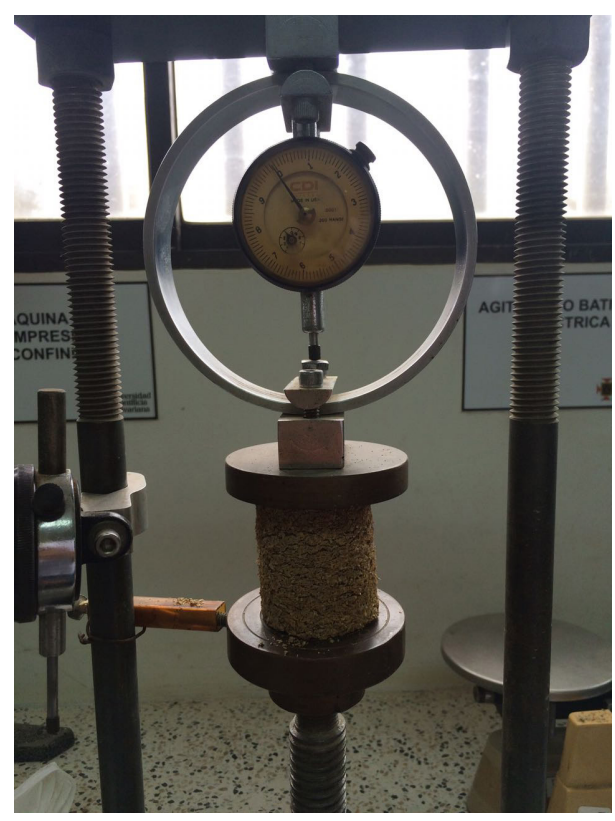

Figura 3. Prueba de Resistencia a la compresión. Fuente: Autores (2015). Registro fotográfico.

Se calcularon el poder calorífico y el porcentaje de azufre de cada mezcla teniendo en cuenta los aportes energéticos y contenidos de azufre de cada componente que la componen, obtenidos por medio de un análisis elemental y próximo de la biomasa y el carbón mineral.

Para el poder calorífico se realiza la sumatoria de los productos entre los aportes energéticos de cada factor (Biomasa, carbón y aglomerante), y el contenido de estos elementos en la mezcla, como se nota en la ecuación (1).

$$
\begin{aligned}
& P C_{T}=[15,88 * B(\%)]+[23,93 * C(\%)]+ \\
& {[10,34 * A g(\%)]}
\end{aligned}
$$

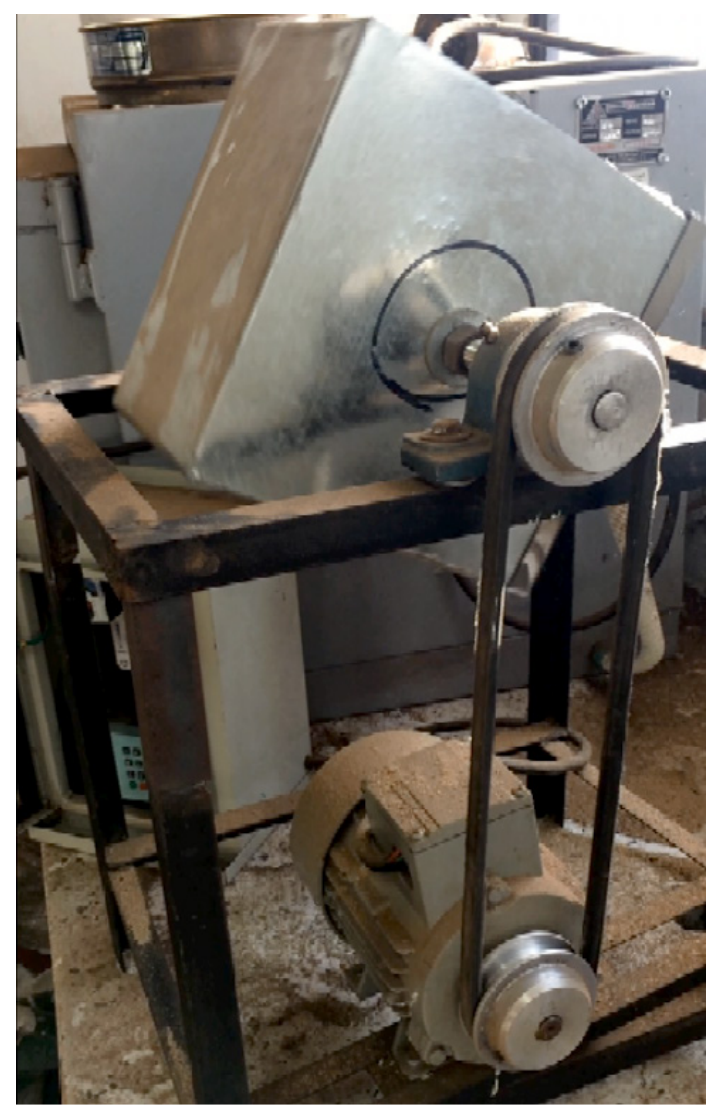

Figura 4. Máquina de durabilidad.

Fuente: Autores (2015). Registro fotográfico.

Donde:

$P C=$ Poder calorífico de cada mezcla.

$B(\%)=$ Porcentaje en masa de biomasa en la mezcla.

$C(\%)=$ Porcentaje en masa de carbón en la mezcla. $A g(\%)=$ Porcentaje en masa de aglomerante en la mezcla.

Análogamente, se calcularon los porcentajes de azufre de cada mezcla, realizando la sumatoria de los productos del porcentaje de azufre de cada factor y el contenido de estos en la mezcla como se muestra en la ecuación (2).

$$
\begin{aligned}
& \% S_{T}=[0,09 * B(\%)]+[1,55 * C(\%)]+ \\
& {[0 * A g(\%)]}
\end{aligned}
$$

Dónde:

$\% S \quad=$ Contenido de azufre de cada mezcla. 
Los resultados obtenidos en las pruebas anteriores, el poder calorífico y porcentaje de azufre de cada corrida, calculados mediante un método determinístico, se tabularon y fueron analizados, en la versión 9 del Software Design Expert por medio de un modelo simplex reticular, debido a su capacidad para determinar el comportamiento de un experimento con tres factores, generando modelos de regresión experimental para la resistencia a la compresión y la durabilidad que, a su vez, fueron usados por el método de superficie de respuesta (RMS) para optimizar las mezclas mediante la interacción de los factores del modelo y la minimización o maximización de una función multiobjetivo, ajustada a una determinada deseabilidad en torno a los factores y variables de respuesta.

\section{RESULTADOS}

De acuerdo con los límites establecidos en el porcentaje de los factores, se obtuvo la siguiente batería de ensayos, además de los resultados de las pruebas de resistencia a la compresión y durabilidad, junto a los porcentajes de azufre y poder calorífico calculados para cada mezcla aplicando un método determinístico.

Respecto a la durabilidad se puede concluir, que las mezclas T1, T5, T7, T9, T12 y T15 lograron sobrepasar un porcentaje de $90 \%$, que según la norma corresponde a valores aceptables de durabilidad, siendo 97,3\% el valor más alto de las briquetas, alcanzado por la mezcla T7. De igual forma, según los resultados de resistencia a la compresión, se encontró que el $75 \%$ de las mezclas superó un valor de $70 \mathrm{~kg}_{\mathrm{f}}$ necesarios para briquetas de $50 \mathrm{~g}$, según la norma técnica colombiana NTC-2060 de briquetas combustibles.

Por último, es importante mencionar que mientras aumenta el contenido de carbón en las mezclas, mayor es el poder calorífico de estas; sin embargo, también aumenta el porcentaje de azufre en las briquetas como se nota en las Tablas 2 y 3 de mezclas y de resultados respectivamente.

Modelo de regresión para la durabilidad mecánica: Desarrollado en función del porcentaje en peso de cada uno de estos componentes, ver ecuación (3):

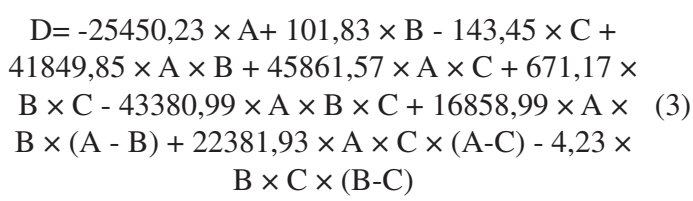

D: Durabilidad.

A: Aglomerante.

B: Biomasa.

C: Carbón.

Fuente: Autores, Design Expert Versión 9 (2015).

Tabla 2. Tabla de mezclas.

\begin{tabular}{|c|c|c|c|c|}
\hline Mezcla & Corridas & Aglomerante (\%) & Biomasa (\%) & Carbón (\%) \\
\hline T1 & 1 & 15 & 85 & 0 \\
\hline T2 & 2 & 20 & 53,33 & 26,67 \\
\hline T3 & 3 & 10 & 50 & 40 \\
\hline T4 & 4 & 8,75 & 61,25 & 30 \\
\hline T5 & 5 & 5 & 68,33 & 26,67 \\
\hline T6 & 6 & 20 & 66,67 & 13,33 \\
\hline T7 & 7 & 10 & 90 & 0 \\
\hline T8 & 8 & 16,25 & 53,75 & 30 \\
\hline T9 & 9 & 8,75 & 81,25 & 10 \\
\hline T10 & 10 & 20 & 40 & 40 \\
\hline T11 & 11 & 15 & 45 & 40 \\
\hline T12 & 12 & 5 & 95 & 0 \\
\hline T13 & 13 & 16,25 & 73,75 & 10 \\
\hline T14 & 14 & 5 & 55 & 40 \\
\hline T15 & 15 & 20 & 80 & 0 \\
\hline T16 & 16 & 5 & 81,67 & 13,33 \\
\hline
\end{tabular}

Fuente: Autores (2015). 
Tabla 3. Tabla de resultados.

\begin{tabular}{|c|c|c|c|c|c|}
\hline Mezcla & Corridas & Poder calorífico (MJ/kg) & Azufre (\%) & Durabilidad (\%) & Resistencia (kgf) \\
\hline T1 & 1 & 15,05 & 0,08 & 95,77 & 223,69 \\
\hline T2 & 2 & 16,92 & 0,46 & 8,56 & 25,53 \\
\hline T3 & 3 & 18,54 & 0,67 & 29,22 & 90,19 \\
\hline T4 & 4 & 17,81 & 0,52 & 77,20 & 215,22 \\
\hline T5 & 5 & 17,75 & 0,47 & 94,76 & 223,69 \\
\hline T6 & 6 & 15,84 & 0,27 & 42,88 & 189,64 \\
\hline T7 & 7 & 15,32 & 0,08 & 97,30 & 223,69 \\
\hline T8 & 8 & 17,4 & 0,51 & 37,54 & 36,35 \\
\hline T9 & 9 & 16,2 & 0,23 & 91,12 & 223,69 \\
\hline T10 & 10 & 17,99 & 0,66 & 7,07 & 13,41 \\
\hline T11 & 11 & 18,27 & 0,66 & 17,44 & 6,51 \\
\hline T12 & 12 & 15,6 & 0,09 & 95,95 & 223,69 \\
\hline T13 & 13 & 15,78 & 0,22 & 83,37 & 132,88 \\
\hline T14 & 14 & 18,82 & 0,67 & 78,21 & 204,26 \\
\hline T15 & 15 & 14,77 & 0,07 & 69,57 & 86,88 \\
\hline T16 & 16 & 16,67 & 0,28 & 96,93 & 223,69 \\
\hline
\end{tabular}

Fuente: Autores (2015).

Análisis de superficie de respuesta para la durabilidad: En la superficie de respuesta y el grafico de contorno para la durabilidad de la tusa de maíz que se muestran en las Figuras 5 y 6 respectivamente. Es notable el hecho de que la durabilidad mecánica de las briquetas aumenta en la medida en que el contenido de biomasa de estas se acerca a su valor máximo (95\%); sin embargo, los

Design-Expert $\otimes$ Software

Component Coding: Actual

Durabilidad (\%)

- Design points above predicted value

Design poir

7.06796

$\mathrm{X} 1=\mathrm{A}:$ Aglomerante

$X_{2}=B$ : Biomasa

$X_{3}=\mathrm{C}:$ Carbón

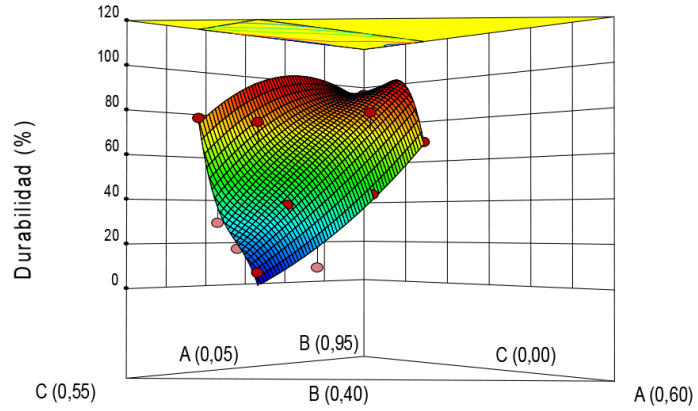

Figura 5. Superficie de respuesta para la durabilidad en briquetas de tusa de maíz.

Fuente: Autores, Design Expert Versión 9 (2015).

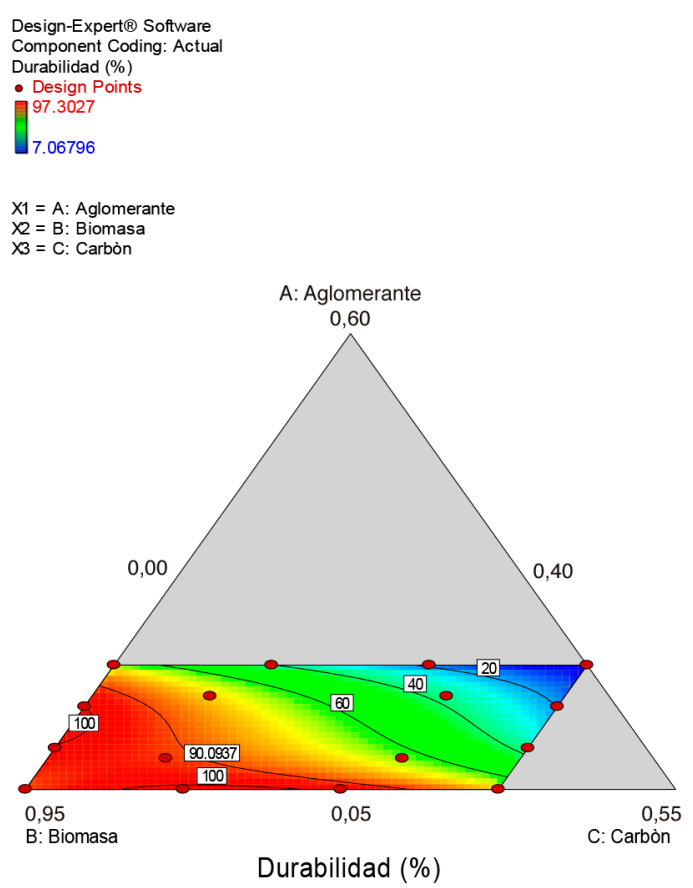

Figura 6. Gráfico de contorno para la durabilidad en briquetas de tusa de maíz.

Fuente: Autores, Design Expert Versión 9 (2015).

mayores valores de durabilidad no se logran en las briquetas con un $95 \%$ de contenido de tusa, si no que estos valores se obtienen a partir de contenidos de 
carbón de $7 \%$ a $25 \%$ y una adición de aglomerante inferior a $6 \%$, lo que indica que se pueden obtener densificados de excelente durabilidad (DU entre 95\% y $100 \%$ ), usando hasta un mínimo de $70 \%$ de biomasa en la mezcla.

Se encontró también que es poco probable obtener briquetas durables (DU mayor a 90\%) usando porcentajes superiores a 32\% de carbón mineral en las mezclas, sin importar que proporciones de biomasa y aglomerante sean utilizados. Análogamente al usar cantidades superiores a un $18 \%$ de aglomerante en la mezcla, no es posible obtener una durabilidad aceptable en los densificados.

Modelo de regresión para la resistencia a la compresión: El modelo de regresión que predice la resistencia a la compresión de las briquetas a base de tusa de maíz aglomerante de yuca y carbón, en función del porcentaje en peso de cada uno de estos componentes, ver ecuación (4):

$\mathrm{R}=71429,08 \times \mathrm{A}-3,37 \times \mathrm{B}+1949,6 \times \mathrm{C}-1,24 \times$

$\mathrm{A} \times \mathrm{B}-1,17 \times \mathrm{A} \times \mathrm{C}-2546 \times \mathrm{B} \times \mathrm{C}+99207,11 \times$

$\mathrm{A} \times \mathrm{B} \times \mathrm{C}-59444,03 \times \mathrm{A} \times \mathrm{B} \times(\mathrm{A}-\mathrm{B})-30651,25$

$\times \mathrm{A} \times \mathrm{C} \times(\mathrm{A}-\mathrm{C})+1528,02 \times \mathrm{B} \times \mathrm{C} \times(\mathrm{B}-\mathrm{C})$

R: Resistencia a la compresión.

Fuente: Autores, Design Expert Versión 9 (2015).

Superficie de respuesta para resistencia a la compresión: Según lo indicado por las Figuras 7 y 8 , al igual que la durabilidad, la resistencia a la compresión de las briquetas hechas a base tusa de maíz, tiene la tendencia a aumentar a medida que el porcentaje de biomasa en la mezcla es mayor. Además, se pudo determinar que siempre será posible obtener una resistencia a la compresión aceptable (mayor que $70 \mathrm{KgF}$, según la norma técnica colombiana NTC-2060 de briquetas combustibles) en las briquetas fabricadas con contenidos de biomasa superiores a $57 \%$, sin importar las concentraciones de aglomerante y carbón entre los límites establecidos para el diseño experimental. De igual manera siempre se conseguirán densificados resistentes, usando cantidades de carbón y aglomerante inferiores a $25 \%$ y $11 \%$ de contenido en la mezcla, respectivamente.

Relación entre la durabilidad y resistencia a la compresión: Un resultado importante se presenta

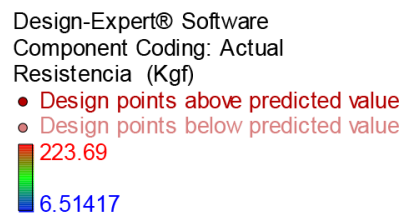

$\mathrm{X} 1=\mathrm{A}:$ Aglomerante

$\mathrm{X} 2=\mathrm{B}$ : Biomasa

X3 = C: Carbón

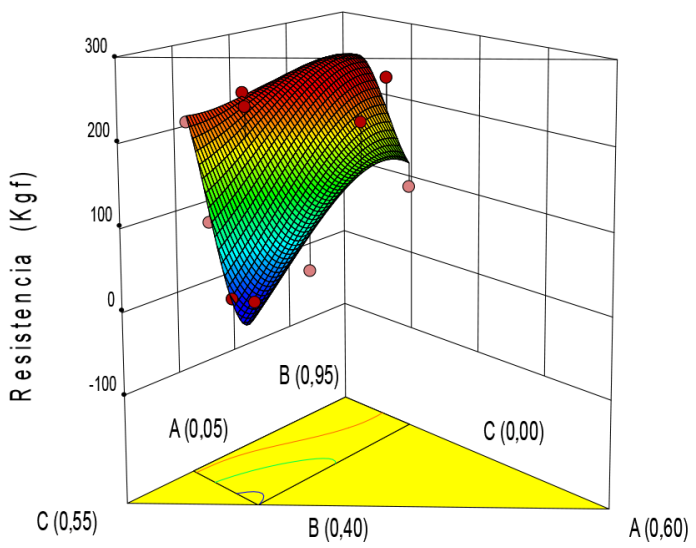

Figura 7. Superficie de respuesta de la resistencia a la compresión en briquetas de tusa de maíz.

Fuente: Autores, Design Expert Versión 9 (2015).

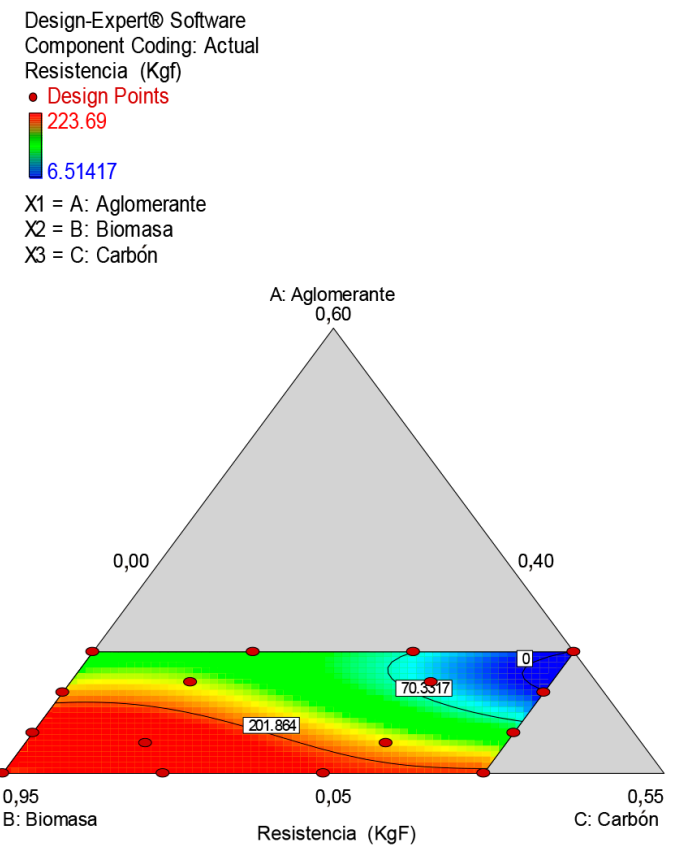

Figura 8. Gráfico de contorno para la resistencia a la compresión en briquetas de tusa de maíz.

Fuente: Autores, Design Expert Versión 9 (2015). 
a partir de la gráfica durabilidad vs. resistencia, generada a partir de los resultados para la tusa de maíz (ver Figura 9), en la cual se puede ver con claridad que hay una relación entre estas dos variables (covarianza positiva), es decir, que si se obtiene una briqueta con una durabilidad mecánica aceptable, muy probablemente esta también cuente con condiciones favorables de resistencia a la compresión; sin embargo, no sucede lo contrario, es decir, una briqueta con una buena resistencia, no garantiza una durabilidad aceptable.

Mezclas óptimas: Para la determinación de las mezclas óptimas de las briquetas a base de tusa de maíz, se tomaron como referencia los comportamientos de las variables de respuesta experimentales frente a variaciones en los factores, además del objetivo inicial de la investigación, los cuales fueron de gran importancia para determinar la deseabilidad del modelo de optimización. De esta manera, se definió minimizar el contenido de aglomerante en la mezcla y maximizar el contenido de biomasa.

Con el fin de aumentar el poder calorífico de las briquetas, por encima del poder calorífico de la biomasa
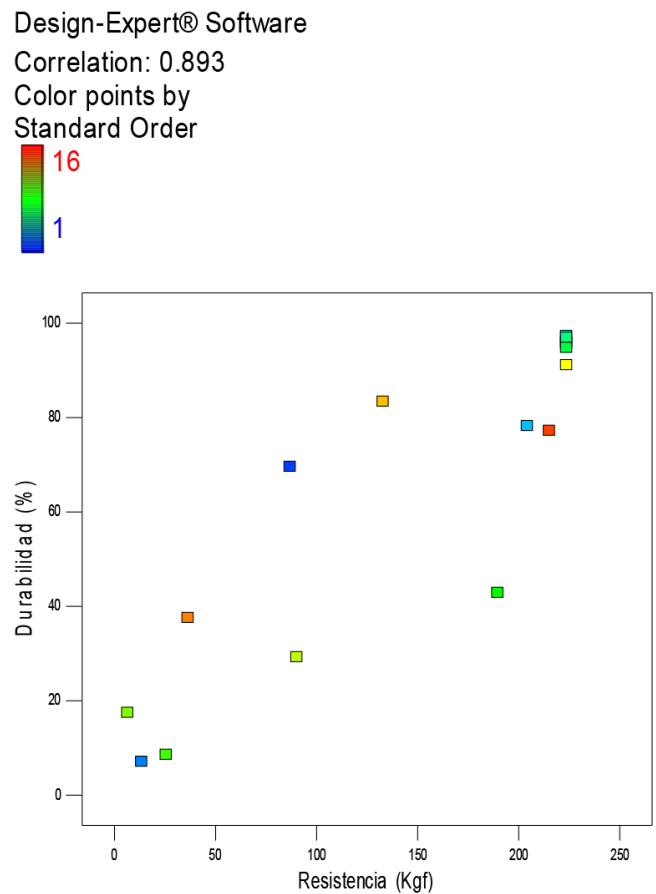

Figura 9. Durabilidad vs resistencia en briquetas de tusa de maíz.

Fuente: Autores, Design Expert Versión 9 (2015). pura $(15,9 \mathrm{MJ} / \mathrm{Kg})$ se decidió maximizar el contenido de carbón, sin embargo se hace necesario controlar el incremento de este factor debido a que también aumenta el porcentaje de azufre de las mezclas, por ello se definió minimizar el porcentaje de azufre de estas.

Por último, se fijó la deseabilidad de la durabilidad y la resistencia en rangos de valores que garantizaron briquetas de calidad según la normatividad (ver Tablas 4a, 4b, 4c, 4d y 4e).

Tabla 4a. Parámetros de optimización 1.

\begin{tabular}{|l|l|c|c|}
\cline { 3 - 4 } \multicolumn{2}{c|}{ Nombre } & Inferior & Superior \\
\hline A: Aglomerante & Minimizar & 0,05 & 0,2 \\
\hline B: Biomasa & Minimizar & 0,4 & 0,95 \\
\hline C: Carbón & Minimizar & 0 & 0,4 \\
\hline Durabilidad & En el rango & 90 & 97,3 \\
\hline Resistencia & En el rango & 70 & 223,69 \\
\hline Poder calorífico & Maximizar & 14,77 & 18,82 \\
\hline$\%$ azufre & Maximizar & 0,07 & 0,67 \\
\hline
\end{tabular}

Fuente: Autores, Design Expert Versión 9 (2015).

Tabla 4b. Parámetros de optimización 2.

\begin{tabular}{|l|c|c|c|}
\cline { 2 - 3 } \multicolumn{1}{c|}{ Nombre } & Inferior & Superior & \multicolumn{1}{c}{} \\
\hline A: Aglomerante & 1 & Peso & Importancia \\
\hline B: Biomasa & 1 & 1 & 3 \\
\hline C: Carbón & 1 & 1 & 3 \\
\hline Durabilidad & 1 & 1 & 3 \\
\hline Resistencia & 1 & 1 & 3 \\
\hline Poder calorífico & 1 & 1 & 3 \\
\hline$\%$ Azufre & 1 & 1 & 3 \\
\hline
\end{tabular}

Fuente: Autores, Design Expert Versión 9 (2015).

Tabla 4c. Mezclas óptimas 1.

\begin{tabular}{|c|c|c|c|c|}
\cline { 1 - 2 } Soluciones & \multicolumn{4}{|c|}{} \\
\cline { 1 - 4 } Numero & $\begin{array}{c}\text { \% } \\
\text { Agl. }\end{array}$ & $\begin{array}{c}\% \\
\text { Biomasa }\end{array}$ & $\begin{array}{c}\text { \% } \\
\text { Carbón }\end{array}$ & $\begin{array}{c}\text { Durabilidad } \\
(\%)\end{array}$ \\
\hline 1 & 0,05 & 0,71 & 0,24 & 97,3 \\
\hline 2 & 0,05 & 0,68 & 0,27 & 97,3 \\
\hline 3 & 0,05 & 0,91 & 0,04 & 96,4 \\
\hline 4 & 0,11 & 0,79 & 0,1 & 90 \\
\hline 5 & 0,14 & 0,79 & 0,07 & 90 \\
\hline
\end{tabular}

Fuente: Autores, Design Expert Versión 9 (2015). 
Tabla 4d. Mezclas óptimas 2.

\begin{tabular}{|c|c|c|}
\hline Soluciones & & \\
\hline Número & $\begin{array}{l}\text { Resistencia } \\
\text { (Kgf) }\end{array}$ & $\begin{array}{c}\text { Poder calorífico } \\
(\mathrm{MJ} / \mathbf{K g})\end{array}$ \\
\hline 1 & 223,7 & 17,5 \\
\hline 2 & 219,7 & 17,76 \\
\hline 3 & 223,7 & 15,92 \\
\hline 4 & 223,7 & 16,021 \\
\hline 5 & 193,05 & 15,64 \\
\hline
\end{tabular}

Tabla 4e. Mezclas óptimas 3.

\begin{tabular}{|c|c|c|}
\hline Soluciones & \multicolumn{2}{|c|}{} \\
\hline Número & \% azufre & Deseabilidad \\
\hline 1 & 0,43 & 0,61 \\
\hline 2 & 0,5 & 0,6 \\
\hline 3 & 0,143 & 0,47 \\
\hline 4 & 0,22 & 0,47 \\
\hline 5 & 0,18 & 0,4 \\
\hline
\end{tabular}

Según los criterios de deseabilidad establecidos, fueron halladas cinco posibles soluciones, donde el software recomienda seleccionar la mezcla número 1. No obstante, dependiendo de los resultados que se quieran obtener, es posible elegir cualquiera de ellas. Es de recalcar el hecho de que la mezcla número 5 no supera en poder calorífico a la biomasa pura, por lo que energéticamente no cumple con el objetivo, sin embargo; esta mezcla, logra mantener el 98,5\% del poder calorífico de la biomasa pura, además de poseer un muy bajo porcentaje de azufre en comparación con las demás mezclas, de igual forma, la 3 y 4 , no lograron un aumento significativo en el poder calorífico, siendo este de $0,27 \%$ y $0,9 \%$ respectivamente.

Todas las soluciones tienen valores de sus propiedades mecánicas dentro de los considerados aceptables para briquetas, ahora bien, en términos energéticos la mezcla 1 y 2 presentaron un aumento importante en cuanto a poder calorífico de $10,25 \%$ y $11,85 \%$, respectivamente, producto de un mayor porcentaje de carbón en la mezcla; sin embargo, este mayor aumento en el contenido de carbón, también representa un mayor porcentaje de azufre, aunque ninguna de las dos soluciones supere los limites en contenido de azufre, planteados por normativas ambientales según la resolución 623 de 1998 del Ministerio de Ambiente colombiano, con una vigencia a partir del
2006, los carbones y sus mezclas que se utilicen en la costa atlántica como combustible, deberán cumplir con un porcentaje de azufre inferior al $1 \%$. En consecuencia, según los criterios establecidos en este estudio, fueron seleccionadas las mezclas 1 y 2 como óptimas, ya que presentaron los mejores resultados en cuanto a durabilidad y resistencia a la compresión, además de un aumento significativo del poder calorífico de la mezcla y un porcentaje de azufre dentro de la normatividad (ver Tabla 4b).

\section{CONCLUSIONES}

Se lograron elaborar biocombustibles sólidos densificados (briquetas), a partir de tusa de maíz, aglomerante de yuca y carbón mineral.

El 37,5\% de las mezclas de tusa de maíz, obtuvieron una durabilidad superior al $90 \%$ y el $75 \%$ una resistencia a la compresión mayor a $70 \mathrm{Kgf}$.

De manera general, el carbón mineral actúa como un factor disociador en las mezclas, reduciendo considerablemente la durabilidad y resistencia a la compresión en las briquetas.

No es posible obtener briquetas durables con porcentajes superiores a $32 \%$ de carbón mineral y $18 \%$ de aglomerante en la mezcla.

Si se obtiene una briqueta con más de $225 \mathrm{Kgf}$, es muy probable que esta tenga una durabilidad mayor a $90 \%$.

Los mayores valores de durabilidad (95-100\%) se logran usando carbón en porcentajes de 7 a $25 \%$, aglomerante menor a $6 \%$ y un contenido de biomasa de hasta $70 \%$.

Se encontraron las mezclas óptimas, para briquetas a base de tusa de maíz, con porcentaje de aglomerante de $5 \%$, biomasa de $68 \%$ a $71 \%$ y carbón mineral de 24 a 27\%, asimismo, se logró aumentar el poder calorífico de la briqueta hasta en $11,85 \%$ respecto al de la biomasa pura, conservando buenas propiedades de durabilidad y resistencia a la compresión, además de un porcentaje de azufre de $0,3 \%$.

\section{AGRADECIMIENTOS}

Los autores de este trabajo desean agradecer a la Facultad de Ingeniería Mecánica de la Universidad 
Pontificia Bolivariana Montería, y al aporte de recursos del convenio 753 de 2013 "Desarrollo de Integración Tecnológica de Recursos Energéticos Renovables en Sistemas Productivos Agrícolas y Agroindustriales Montería, Córdoba, Caribe", BPIN No 2012000100026.

\section{REFERENCIAS}

[1] Departamento Administrativo Nacional de Estadística. "Resultado Encuesta Nacional Agropecuaria ENA". DANE. 2011. URL: http://www.dane.gov.co/files/investigacion es/agropecuario/enda/ena/doc_anexos_ ena_2011.pdf

[2] L.O. Torres. "Producción de Biocombustibles Sólidos de Alta Densidad En España". Universidad de Vigo. España. 2008.

[3] J.P. Sánchez. "Los Bosques como sistema de Bioalmacenamiento y Bioenergía". Universidad de Oviedo. España. 2012.

[4] N. Kaliyan. "Densification of Biomass: Mechanisms". Models, and Experiments on Briquettingand Pelleting of Biomass. March, 2008.

[5] F.M. Martín. "Pélets y briquetas". AITIM - Asociación de Investigación Técnica de las Industrias de la Madera. Vol. 171, pp. 54-62. 1994.

[6] J.M. Stolarski, S. Szczukowski, J. Tworkowski, M. Krzyżaniak, P. Gulczyński and M. Mleczek. "Comparison of quality and production cost of briquettes made from agricultural and forest origin biomass". Renewable Energy. Vol. 57, pp. 20-26. 2013.
[7] T. Ivanova, M. Kolarikova, B. Havrland, and L. Passian. Mechanical durability of briquettes made of energy crops and wood residues. Engineering for rural development, pp. 131-136. 2014.

[8] G. Soto y M. Núñez. "Fabricación de pellets de carbonilla, usando aserrín de pinus radiata (D. Don), como material aglomerante". Maderas, Ciencia y Tecnología. Vol. $10 \mathrm{~N}^{\circ} 2$, pp. 129-137. 2008.

[9] J.T. Oladeji. "Fuel Characterization of Briquettes Produced from Corncob and Rice Husk Resides". Ladoke Akintola University of Technology. Ogbomoso, Nigeria. 2010.

[10] M. Temmerman, F. Rabier, P.D. Jensen, H. Hartmann and T. Böhm. "Comparative study of durability test methods for pellets and briquettes". Biomass and Bioenergy, Vol. 30, Issue 11, pp. 964-972. 2006.

[11] J. Zhang and Y. Guo. "Physical properties of solid fuel briquettes made from Caragana korshinskii Kom". Powder Technology. Vol. 256, pp. 293-299. April. 2014.

[12] L. Wamukonya and B. Jenkins. "Durability and relaxation of sawdust and wheat-straw briquettes as possible fuels for Kenya". Biomass and Bioenergy. Vol. 8, Issue 3, pp. 175-179. Elsevier. 1995.

[13] F.C. Edison y T.T. Fernando. "Desarrollo de un proceso tecnológico para la obtención de briquetas de aserrín de madera y cascarilla de arroz, y pruebas de producción de gas pobre". Escuela Superior Politécnica de Chimborazo, Riobamba, Ecuador. 2011. 